\title{
Treatment of chronic limb spasticity with botulinum toxin $\mathrm{A}$
}

\author{
John W Dunne, Norbert Heye, Sandra L Dunne
}

\begin{abstract}
The purpose of this open study was to find out whether botulinum toxin $A$ (BTX-A) relieves the signs and symptoms of chronic limb spasticity. The study comprised 40 patients, aged 12-82 years, with moderate to severe spasticity of the upper (13) or lower limbs (27) refractory to conventional physical and medical treatments. Outcome measures were clinical and blinded videotape assessments of spasticity and motor function. Electromyography guided BTX-A injections were given in one or two sessions at total doses averaging $175 \mathrm{U}$ in the upper limb (range 70-270 $\mathrm{U}$ ) and $221 \mathrm{U}$ in the lower limb (range 100-500 U). Thirty four patients $(85 \%)$ derived worthwhile benefit, with improved limb posture and increased range of passive motion in 31, pain reduction in 28 of 31 with pain, and improved function in 16. Side effects were limited to local and usually mild discomfort from the injections (19), symptomatic local weakness (one), and local infection (one).

Preliminary experience indicates that BTX-A is a promising adjunctive treatment for selected patients with spasticity.
\end{abstract}

$(\mathcal{F}$ Neurol Neurosurg Psychiatry 1995:58;232-235)

Keywords: spasticity; botulinum toxin

Spasticity is a velocity dependent increase in muscle resistance to stretch. The term is often extended to include the associated features of exaggerated tendon reflexes, clonus, and flexor spasms, ${ }^{12}$ and it is in this context that we use it.

The mainstay of treatment remains physiotherapy, but moderate to severe spasticity often responds only partially. Pain, disturbed postures, and permanent contractures may result. Antispasticity medications and the more invasive measures of orthopaedic surgery, neural blockade with alcohol or phenol, and intrathecal baclofen have their limitations.

Botulinum toxin A (BTX-A) produces dose related weakness of skeletal muscle by impairing the release of acetylcholine at the neuromuscular junction. Although shown to be a safe and effective treatment for dystonia, ${ }^{3}$ so far few studies have explored the potential of BTX-A in the treatment of spasticity. ${ }^{4-9}$

The purpose of this open study was to find out whether BTX-A relieves the signs and symptoms of chronic limb spasticity that were refractory to other treatments.

\section{Methods}

Patients were recruited from neurology outpatients or inpatients of the Royal Perth and Royal Perth Rehabilitation Hospitals, after having given their informed consent to participation. Subject entry criteria were moderate to severe spasticity (based on the modified Ashworth spasticity scale) of at least six months' duration with a static or slowly progressive course; refractoriness to conventional physical and medical treatments; specific target muscle(s) enabling practical treatment by local injections; and no change in antispasticity treatment over the trial period. Exclusion criteria were major contractures of target muscles; other chronic disease causing major physical disability in the target region(s) (for example, rheumatoid arthritis); and pregnancy or nursing mothers.

Each patient was assessed by an experienced neurophysiotherapist, a neurologist, and a blinded medical observer, who viewed randomly arranged videotapes performed with a standard protocol. Two baseline assessments were performed three to four weeks apart primarily to ensure stability of signs, but also to monitor the reliability of the assessment procedures. At least two further assessments were performed, four to six weeks and four to six months after injections.

Outcome measures were the modified Ashworth spasticity and spasm frequency scales, ${ }^{5}$ Lindmark's modified motor assessment system, ${ }^{1011}$ measurements of joint angles, a patient pain score, and the Barthel index. The motor assessment system, a modification of the Fugyl-Meyer assessment, is a comprehensive motor function scoring system of all limbs that includes active movements, repetitive movements, and mobility. ${ }^{10}$ Joint angles were assessed by goniometer measurements ${ }^{12}$ and by standardised videotape assessments (posture at rest, range of active 
and passive joint motion). A goniometer measurement change $>5^{\circ}$ was required to indicate change between assessments. ${ }^{12}$ The videotapes of each patient were rated by a five point scale $(0=$ limb rigid in flexion, $1=$ pronounced restriction, $2=$ moderate restriction, $3=$ mild restriction, $4=$ normal). Pain related to flexor spasms or passive stretch was rated by the patient on a four point scale $(0=$ no pain to 3 = severe pain), where a change of one point or more was considered substantial. Patient ratings of painful side effects of the procedure used the same scale. The Barthel index of functional independence does not specifically measure limb motor function but it was recorded for completeness. This number of outcome assessments was justified by the wide range of patient disabilities and varying indications for treatment. Validity and reliability have been established for most of these measures. ${ }^{10-12}$

Botulinum toxin A (Botox ${ }^{\circledR}$; Allergan Inc, USA) was diluted to $20 \mathrm{U} / \mathrm{ml}$ and injected into selected muscles under electromyographic (EMG) guidance by one of us (JD), who did not measure outcome. Muscle selection was based primarily on clinical assessment and aimed to improve limb posture and range and ease of passive movement or limb function (by allowing the antagonist muscles to act with less opposition), or to relieve pain. A 25-27 gauge needle was used to record EMG activity and inject in a fan-like field at two to four sites per muscle, targeting the middle third of the muscle belly. The initial dosage of BTX-A varied with individual muscles and the degree of their involvement as judged clinically and as confirmed by EMG. Where response was considered inadequate by JD, supplementary injections were given three to four weeks later. All other elements of the patient's care, including the nature and intensity of physical and occupational therapy, were unaltered.

We compared the baseline measurements with those found after treatment in all patients. The tests of statistical significance were the paired $t$ test for goniometer measurements, the Wilcoxon signed rank sum test for the other ordinal rating scales, and Kendell's rank correlation for the agreement between the baseline assessments. Significance was at the $1 \%$ level because of the number of comparisons made.

\section{Results}

Forty patients (23 male, 17 female) aged 12-82 (mean 50) years were enrolled, and none withdrew. Their neurological diagnoses were stroke (19), heredofamilial spastic paraparesis (six), multiple sclerosis (four), Friedreich's ataxia (three), hypoxic encephalopathy (two), cerebral palsy (two), motor neuron disease (two), head injury (one), and spinal cord injury (one).

The mean duration of spasticity was 10 (range 0.5-45) years, and for the stroke subgroup it was 3.9 (range $0.5-11$ ) years. All patients were having physiotherapy at least once a week. Other past or ongoing treatments included various antispasticity medications (36), orthotics and splints (22), tenotomies (nine), phenol nerve blocks (three), and intrathecal baclofen pump (one). The target was the upper limb in 13 patients (elbow/forearm five; wrist five; fingers three) and lower limb in 27 (hip 11; ankle nine; toes four; knees three). Upper limb injections were unilateral, and lower limb injections were unilateral in 16 and bilateral in 11 (hip adductors or ankle plantarflexors).

The reasons for treatment were disabling limb posture in 39 patients (for example, elbow flexion, ankle plantarflexion) and painful muscle spasms or painful and difficult passive stretches in 30. Mean total BTX-A dosage for the upper limb was 175 (range 70270) U, for a single lower limb 221 (100-500) $\mathrm{U}$, and for both lower limbs 321 (190-610) $\mathrm{U}$. The number of muscles injected varied from two to six per limb depending on the problem. Only three patients received a total dosage greater than $300 \mathrm{U}$, and the maximum dosage given at a single session was $320 \mathrm{U}$.

Table 1 summarises the results at the two baseline assessment points and at four to six weeks after the last BTX-A injections. There was excellent agreement between the two baseline assessments; Kendell's correla-

Table 1 Outcome measures at the treatment sites: at baseline and after treatment

\begin{tabular}{|c|c|c|c|c|}
\hline Measure & Baseline 1 & Baseline 2 & Post-treatment & $p$ Value \\
\hline \multicolumn{5}{|l|}{ Modified Ashworth spasticity scale (score $0-4$ ): } \\
\hline Spasm frequency scale (score $0-4$ ): & $3.6(0.6)$ & $3.8(0.5)$ & $1.4(0.8)$ & - 00000 \\
\hline \multirow{3}{*}{$\begin{array}{l}\text { Modified motor assessment (parts A-C): } \\
\text { Part A: active movement subscale (involved limb) } \\
\text { Upper limb (score 0-57) } n=13 \\
\text { Lower limb (score 0-36) } n=27 \\
\text { Part B: rapid movement subscale (score 0-12): }\end{array}$} & $50(000)$ & $200(0.7)$ & $1.4(0.8)$ & $<0.0001$ \\
\hline & $\begin{array}{l}5 \cdot 4(6 \cdot 1) \\
8 \cdot 9(6 \cdot 7)\end{array}$ & $\begin{array}{r}6 \cdot 3(5 \cdot 9) \\
10 \cdot 1(7 \cdot 1)\end{array}$ & $\begin{array}{r}8 \cdot 0(7 \cdot 9) \\
12 \cdot 1(7 \cdot 7)\end{array}$ & $\begin{array}{l}\text { NS } \\
0.004\end{array}$ \\
\hline & $3 \cdot 7(2 \cdot 6)$ & $3.7(2 \cdot 7)$ & $3 \cdot 7(2 \cdot 7)$ & NS \\
\hline \multirow[t]{2}{*}{$\begin{array}{l}\text { Part C: mobility subscale (score } 0-27 \text { ): } \\
\text { Upper limb treated } n=13 \\
\text { Lower limb treated } n=27 \\
\text { Passive range of joint motion (goniometer): }\end{array}$} & $\begin{array}{l}16 \cdot 5(9 \cdot 3) \\
14 \cdot 4(9 \cdot 6)\end{array}$ & $\begin{array}{l}16 \cdot 6(9 \cdot 3) \\
14 \cdot 4(9 \cdot 7)\end{array}$ & $\begin{array}{l}16 \cdot 6(9 \cdot 3) \\
17 \cdot 6(8 \cdot 3)\end{array}$ & $\begin{array}{l}\text { NS } \\
0 \cdot 007\end{array}$ \\
\hline & $78(53)$ & $80(52)$ & $110(49)$ & $<0.0001$ \\
\hline \multirow{2}{*}{$\begin{array}{l}\text { Blinded videotape assessment (score } 0-4 \text { ): } \\
\text { Active range } \\
\text { Passive range } \\
\text { Joint pain scale (score } 0-3) n=31 \text { : }\end{array}$} & $\begin{array}{l}0.9(0.9) \\
1.7(1 \cdot 0)\end{array}$ & $\begin{array}{l}0.9(1.0) \\
1.6(0.9)\end{array}$ & $\begin{array}{l}1.4(1.2) \\
2 \cdot 9(0.9)\end{array}$ & $\begin{array}{l}0.0002 \\
<0.0001\end{array}$ \\
\hline & $2.5(0.5)$ & $2.5(0.5)$ & $0.8(0.7)$ & $<0.0001$ \\
\hline
\end{tabular}

Values are mean (SD). 
tion coefficients were $0 \cdot 79-1 \cdot 0(p<0 \cdot 004)$. Improvements after treatment were seen in most outcome measures, these all being related to changes in the treated muscles. Overall a worthwhile benefit occurred in 34 patients $(85 \%)$, with an increased range of passive motion in 31 , pain reduction in 28 of 31 with pain, and improved function in 16 .

The mean change in the modified Ashworth spasticity score at the site of treatment was $1 \cdot 2$ (95\% confidence interval (95\% CI) $1 \cdot 0-1 \cdot 4)$ and in the spasm frequency scale it was $2 \cdot 4(95 \%$ CI $2 \cdot 0-2 \cdot 7)$. Lindmark's modified motor assessment system showed changes confined to the injected limb, so table 1 shows the subscores of a treated upper or a lower limb. Improved active movement subscores in the lower limbs influenced personal hygiene, transfers, and walking. Overall, upper limb active movement subscores did not change significantly $(p=$ 0.06 ), although some had improvements in hand, forearm, and elbow movements. Mobility subscore changes were largely confined to patients having lower limb injections. Treatment enabled a return to walking in five patients and improved gait pattern in 10 , related variously to reduced spasticity in hip adductors, hamstrings, ankle plantarflexors and invertors, or toe flexors. Three of the five ambulant patients with heredofamilial spastic paresis had an improved gait pattern after bilateral hip adductor or ankle plantarflexor and invertor injections. Range of passive joint movement showed a mean improvement of $28^{\circ}\left(95 \%\right.$ CI $\left.21^{\circ}-36^{\circ}\right), 30$ patients showing $a \geqslant 15^{\circ}$ gain. Benefits were comparable at the differing target sites.

Blinded videotape assessments showed at least a 1 grade improvement in passive range of joint motion in 30 patients $(75 \%)$ with a 2-3 grade functional improvement in 16 patients. Comparison of goniometer and videotape assessments of range of passive movement showed a strong and ordered relation ( $p=0.002$, one way analysis of variance).

Pain related to painful flexor spasms (eight) or passive stretching (23) was present before treatment in 31 patients. At least moderate relief occurred in $90 \%, 26 \%$ with complete resolution of pain.

The mean baseline Barthel score was 13 of $20 \quad(29$ patients had a score $\leqslant 14)$, indicating at least moderate functional disabilities in most patients. Although three patients showed improvements $\geqslant 9$ points, overall no change in functional independence occurred (mean change in score 1.3, $\mathrm{p}=0.06)$.

The six patients not responding substantially had no unifying characteristics. The usual time of onset of change was four days, with peak effect at three weeks and a mean duration of five months (range two to 12 months). An EMG of injected muscles in 33 patients at follow up showed moderate fibrillation potentials in all and, in those where this was assessable, EMG showed reduced recruitment.

All 34 patients assessed as having worth- while improvement reported substantial subjective benefit, and wished to have the treatment again if necessary. So far 22 patients have had repeat injections on up to six occasions, spaced by a minimum of four months. A reproducible degree and course of improvement consistent with a BTX-A effect occurred in all.

Adverse effects occurred in 19 patients. Pain during injections was rated as mild by 12 patients and moderate to severe by seven. Symptomatic weakness of injected muscles occurred in one patient (elbow flexion); this was mild and resolved after 10 days. Local infection at a calf injection site occurred in one patient with chronic venous insufficiency. No major or systemic side effects occurred, including the three patients receiving $>300 \mathrm{U}$ (500-610 U over two sessions) and the two patients with motor neuron disease who received 250 and $300 \mathrm{U}$ for extremely painful leg flexor spasms.

\section{Discussion}

We have found that BTX-A can suppress the symptoms and signs of spasticity in selected patients by improving limb posture and range of passive movement, allowing more comfortable limb positioning, improving ease of passive stretch, relieving pain, and in some patients producing functional benefits. Whereas upper and lower limb injections showed comparable changes in most outcome measures, improvements in active movements and mobility were seen mainly after lower limb injections, where walking was influenced in particular.

Overall, 34 patients (85\%) had a worthwhile benefit. In all cases this was directly attributable to weakening of the muscle injected, although another mechanism such as selective effects on fusimotor fibres may have contributed. Improvement occurred irrespective of the underlying cause of spasticity. Even the two patients with motor neuron disease had worthwhile benefit without side effects. Likewise the degree of benefit did not correlate with the duration of spasticity; indicating that BTX-A can be used to treat longstanding spasticity as long as fixed contractures have been avoided.

This study has the limitations of any open trial. Whereas we have considered observer bias with blinded videotape assessments, the patient was not blinded to treatment. The promise of this study is that most of these patients, refractory to other treatments, benefited substantially. All improvements were confined to target muscles, and spasticities of untreated regions in the same and other limbs were unchanged from the baseline measurements. The EMG findings and time course of benefit were consistent with a BTX-A effect, and the benefit has been reproducible in the 22 patients who have had repeat treatment so far. It is important to emphasise that the patients treated were carefully selected, based on (a) the presence of specific and localised target muscles allowing this intervention to be 
Table $2 B T X-A$ dosage range for individual muscles

\begin{tabular}{lc}
\hline Muscle & Dosage (U) \\
\hline Biceps brachii (8) & $25-100$ \\
Brachialis (5) & $25-100$ \\
Brachioradialis (9) & $15-80$ \\
Pronator teres (4) & $20-55$ \\
Flexor digitorum superficialis (8) & $20-150$ \\
Flexor digitorum profundus (7) & $30-120$ \\
Flexor carpi radialis (5) & $20-75$ \\
Flexor carpi ulnaris (3) & $25-60$ \\
Adductor pollicis/thenar (3) & $10-15$ \\
Flexor pollicis longus (2) & $10-20$ \\
Hip adductors (12) & $80-160$ \\
Hamstrings (12) & $40-200$ \\
Iliopsoas (3) & $40-70$ \\
Tibialis posterior (10) & $40-150$ \\
Flexor digitorum longus (8) & $40-125$ \\
Flexor digitorum brevis (5) & $20-70$ \\
Flexor hallucis longus (6) & $25-50$ \\
Flexor hallucis brevis (4) & $15-25$ \\
Tibialis anterior (5) & $30-70$ \\
Triceps surae (10) & $100-240$ \\
\hline No of patients in parenteses.
\end{tabular}

No of patients in parentheses.

practical, and (b) consideration of the potentially adverse functional consequences of weakening certain muscles. In addition, the treatment was an adjunct to rather than a replacement for ongoing physical therapy.

We have used EMG guided delivery of BTX-A to enhance the accuracy of injections, widely infiltrating the muscle at multiple sites. The optimal delivery technique is unknown but recent evidence supports this approach. ${ }^{13-15}$ Our use of a high dilution of 20 $\mathrm{U} / \mathrm{ml}$ may have disadvantages in smaller muscles, but it maximises diffusion and the area of paralysis in larger muscles ${ }^{15}$ and limits wastage. The dosages used for individual muscles (table 2) have been refined by experience with titration for the 40 patients, including their repeat treatments. Our mean period of follow up since enrolment in the study is 1.4 years (range $0.5-3$ years), so we cannot comment on the long term efficacy of BTX-A. Cost (\$Aus 450 per $100 \mathrm{U}$ ampoule), the need for repeat injections, and the potential for development of immunity in some ${ }^{3}$ will restrict the application of BTX-A, and whether it is cost effective when compared with other neural ablative procedures is unknown.
In conclusion, BTX-A has promise as an adjunctive treatment for selected patients with spasticity refractory to physical and medical treatments. Benefit usually lasts from two to six months and side effects are minor and transient, but its role in long term treatment of spasticity and cost effectiveness will require further study.

We thank Dr M W Hayes for his assistance in assessment of patients, and the neurophysiotherapists of Royal Perth Rehabilitation Hospital and Drs G Hankey, R Edis, and E G Stewart-Wynne for referring their patients.

Dr Heye was supported by the Medical Research Fund of Western Australia.

1 Editorial. Spasticity. Lancet 1989;ii:1488-90.

2 Young RR, Delwade PJ. Drug therapy: spasticity (first of two parts). N Engl F Med 1981;304:28-33.

3 Clinical use of botulinum toxin A. National Institutes of Health consensus development statement. Arch Neurol 1991;48:1294-7.

4 Das TK, Park DM, Collings AD. Botulinum toxin in treating spasticity. Br 7 Clin Pract 1989;43:401-3.

5 Tsui JKC, O'Brian CF. Clinical trials for spasticity. In: Jankovic J, Hallett $M$, eds. Therapy with botulinum toxin. 1st ed. New York: Marcel Dekker, 1994

6 Dengler R, Neyer U, Wohlfahrt K, Bettig U, Kanzik HH. Local botulinum toxin in the treatment of spastic foot drop. F Neurol 1992;239:375-8.

7 Mémin B, Pollak P, Hommel M, Perret J. Traitement de la spasticité par la toxine botulique. Rev Neurol 1992; 148:212-4.

8 Koman LA, Mooney JF, Smith B, Goodman A, Mulvaney T. Management of cerebral palsy with botulinum-A toxin: preliminary investigation. $\mathcal{f}$ Pediatr Orthop 1993; 13:489-95.

9 Benecke R. Botulinum toxin for spasms and spasticity in the lower extremities In: Jankovic J, Hallett $\mathbf{M}$, eds. Therapy with botulinum toxin. 1st ed. New York: Marcel Dekker, 1994:557-66.

10 Lindmark B, Hamrin E. Evaluation of functional capacity after stroke as a basis for active intervention. Presentation of a modified chart for motor capacity assessment and its reliability. Scand $\mathfrak{f}$ Rehabil Med assessment and

11 Lindmark B, Hamrin E. Evaluation of functional capacity after stroke as a basis for active intervention. Validation of a modified chart for motor capacity assessment. Scand $\mathscr{f}$ Rehabil Med 1988;20:111-5.

12 Boone DC, Azen SP, Lin C-M, Spence C, Baron C, Lee L. Reliability of goniometric measurements. Physical Therapy 1978;58:1355-60.

13 Comella CL. Electromyography-assisted botulinum toxin injections for cervical dystonia. In: Jankovic J, Hallett $M$, eds. Therapy with botulinum toxin. 1st ed. New York: Marcel Dekker, 1994:289-98.

14 Borodic GE, Pearce LB, Joseph M, Smith $\mathrm{K}$. Botulinum toxin A for the treatment of spasmodic torticollis: single toxin A for the treatment of spasmodic torticollis: single
versus multiple injections per muscle. Head Neck 1992; versus multi. $143-7$.

15 Shaari CM, Sanders I. Quantifying how location and dose of botulinum toxin injections affect muscle paralysis. Muscle Nerve 1993;16:964-9. 